\title{
Discovery of New Proxyphylline Based Chiral Cocrystals: Solid State Landscape and Dehydration Mechanism
}

\author{
Lina C. Harfouche,$^{\dagger}$ Nicolas Couvrat,${ }^{\dagger}$ Morgane Sanselme,${ }^{\dagger}$ Clément Brandel,,${ }^{\dagger}$ Yohann \\ Cartigny,,$^{\dagger *}$ Samuel Petit, ${ }^{\dagger}$ and Gérard Coquere ${ }^{\dagger \ddagger}$ \\ $\dagger$ Normandie Univ, UNIROUEN, SMS, 76000 ROUEN, FRANCE. \\ ¥ Department of Chemical and Materials Engineering, National Central University, 300 Zhongda Road, Zhongli \\ District, Taoyuan City 32001, Taiwan R.O.C.
}

\section{Content:}

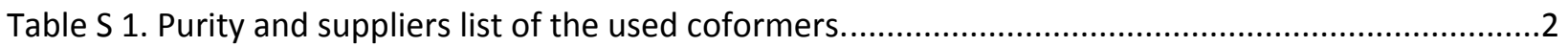

Table S 2. Preparation methods to obtain new cocrystal forms of PXL with six different coformers..........2

Table S 3. SHG activity for the solid forms obtained with 1:1 molar ratio mixtures of (RS)-PXL and different coformers.

Figure S $1 .{ }^{1} \mathrm{H}$ NMR of the cocrystal obtained between PXL and OA in MeOD. Hydrogens of OA were not

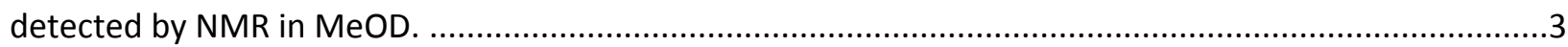

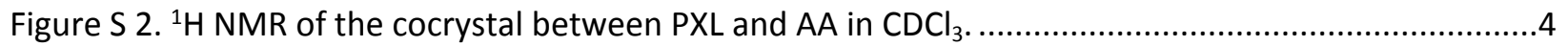

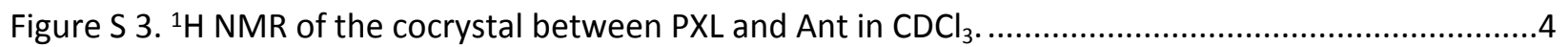

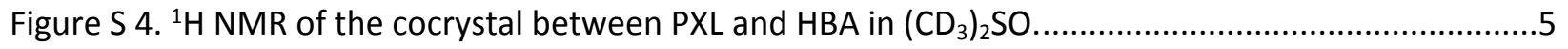

Figure S 5. ${ }^{1} \mathrm{H}$ NMR of the cocrystal between PXL and DMCA in MeOD ..............................................

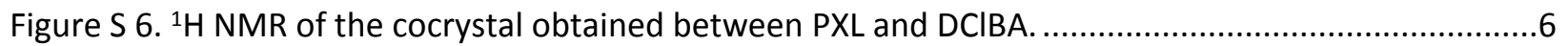

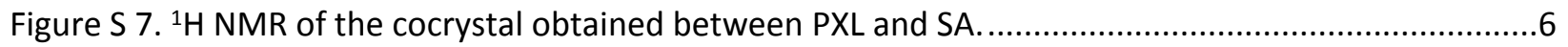

Figure $S$ 8. a) XRPD pattern of the starting material (RS)-PXL, Oxalic acid (OA) and (RS)-PXL:OA cocrystal

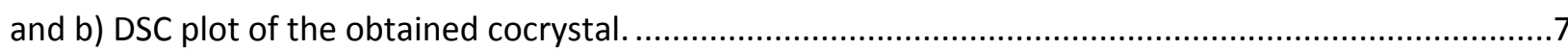

Figure S 9. a) XRPD pattern of (RS)-PXL, AA, E-PXL, (RS)-PXL:AA cocrystal and E-PXL:AA cocrystal in 1:1 molar ratio and b) DSC plot of the obtained cocrystals.......................................................................

Figure S 10. a) XRPD pattern of (RS)-PXL, Ant and their mixture in 1:1 molar ratio. b) DSC plot for the

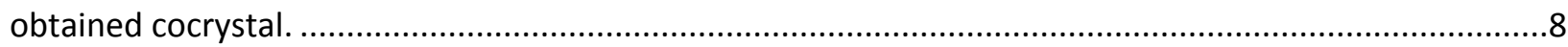

Figure S 11. a) Powder X-ray diffraction for (RS)-PXL, HBA and (RS)-PXL:HBA cocrystal and b) DSC melting curves of the cocrystal.

Figure S 12. a) XRPD for (RS)-PXL, DCIBA and PXL-DCIBA cocrystal and b) DSC melting curve of the new cocrystal form.

Figure S 13. a) XRPD for (RS)-PXL, DMCA and (RS)-PXL-DMCA cocrystal and b) DSC melting curve of the obtained cocrystal.

Figure S 14. Conformational similarity between the PXL molecules from the asymmetric unit of Form- $\mathrm{H}$

(Blue) and Form-A2 (Pink).

Figure $\mathrm{S} 15$. Conformational similarity between the $\mathrm{SA}$ molecules from the asymmetric unit of Form- $\mathrm{H}$ (Blue) and Form-A2 (Pink). 
Figure S 16. Sorption-desorption cycle performed at $25^{\circ} \mathrm{C}$ for SA with DVS vacuum. Mass change (\%) is referred to the mass at the end of the first drying step.

Figure S 17. XRPD patterns of (RS)-A1, (RS)-A2, E-A1 and the obtained solid after annealing (RS)-A1 at

$950 \mathrm{C}$.

Figure S 18. DSC of the anhydrous form A3.

Figure $S$ 19. Experimental and calculated patterns of (E)-H and (RS)-A2 respectively.

Table S 1. Purity and suppliers list of the used coformers.

\begin{tabular}{|c|c|c|}
\hline Coformer & Purity & Supplier \\
\hline 4-methoxybenzoic acid & $>98 \%$ & Alfa Aesar \\
\hline 3-chlorobenzoic acid & $>99 \%$ & Acros Organics \\
\hline 4-dimethylaminobenzoic acid & $>98 \%$ & Acros organics \\
\hline 3-hydroxy-4-nitrobenzoic acid & $>98 \%$ & Acros organics \\
\hline 3,4-dichlorobenzoic acid & $>99 \%$ & Acros organics \\
\hline 2,6-dichlorobenzoic acid & $>98 \%$ & Acros organics \\
\hline Benzamide & $>98 \%$ & Alfa Aesar \\
\hline Urea & $>98 \%$ & VWR Chemicals \\
\hline Adipic acid & $>99 \%$ & Alfa Aesar \\
\hline Saccharin & $>98 \%$ & Acros organics \\
\hline Stearic acid & $>97 \%$ & Acros organics \\
\hline Methyl urea & $>97 \%$ & Acros organics \\
\hline Citric acid & $>98 \%$ & Acros organics \\
\hline Salicylic acid & $>99 \%$ & Acros organics \\
\hline Acetylsalicylic acid & $>99 \%$ & Acros organics \\
\hline Anthranilic acid & $>99 \%$ & Merck \\
\hline Oxalic acid & $>98 \%$ & Alfa Aesar \\
\hline 3,4-dimethoxycinnamic acid & $>99 \%$ & Alfa Aesar \\
\hline 2,5-dichlorobenzoic acid & $>97 \%$ & Acros Organics \\
\hline 3-hydroxybenzoic acid & $>99 \%$ & Acros organics \\
\hline
\end{tabular}

Table S 2. Preparation methods to obtain new cocrystal forms of PXL with six different coformers.

\begin{tabular}{|c|c|c|c|}
\hline Coformer & Neat Grinding & LAG & Evaporation \\
\hline Oxalic acid (OA) & + & $\begin{array}{c}\text { Acetone, IPA, DCM, } \\
\mathrm{CHCl}_{3}\end{array}$ & $/$ \\
\hline Acetylsalicylic acid (AA) & + & $\begin{array}{c}\text { Acetone, heptane, } \\
\text { DCM, MeOH, EtOH }\end{array}$ & MeOH \\
\hline Anthranilic acid (Ant) & + & $\begin{array}{c}\text { Acetone, EtOH, MeOH, } \\
\mathrm{DCM}\end{array}$ & $/$ \\
\hline 3-hydroxybenzoic acid (HBA) & $/$ & $\mathrm{MeOH}$ & $/$ \\
\hline 3,4-dimethoxycinnamic acid (DMCA) & + & $/$ & Heptane \\
\hline 2,5-dichlorobenzoic acid (DClBA) & + & \multicolumn{2}{|}{} \\
\hline
\end{tabular}

+ : co-crystal formation 
/: no new solid phase was identified

Table S 3. SHG activity for the solid forms obtained with 1:1 molar ratio mixtures of (RS)-PXL and different coformers.

\begin{tabular}{|c|c|c|}
\hline Coformer & $\begin{array}{c}\text { SHG } \\
\text { signal }\end{array}$ & $\begin{array}{c}\text { SHG } \\
\text { intensity }\end{array}$ \\
\hline Oxalic acid (OA) & - & 0 \\
\hline Acetylsalicylic acid (AA) & + & 7 \\
\hline Anthranilic acid (Ant) & + & 10 \\
\hline 3-hydroxybenzoic acid (HBA) & + & 5 \\
\hline 3,4-dimethoxycinnamic acid (DMCA) & + & 135 \\
\hline 2,5-dichlorobenzoic acid (DClBA) & + & 2258 \\
\hline
\end{tabular}

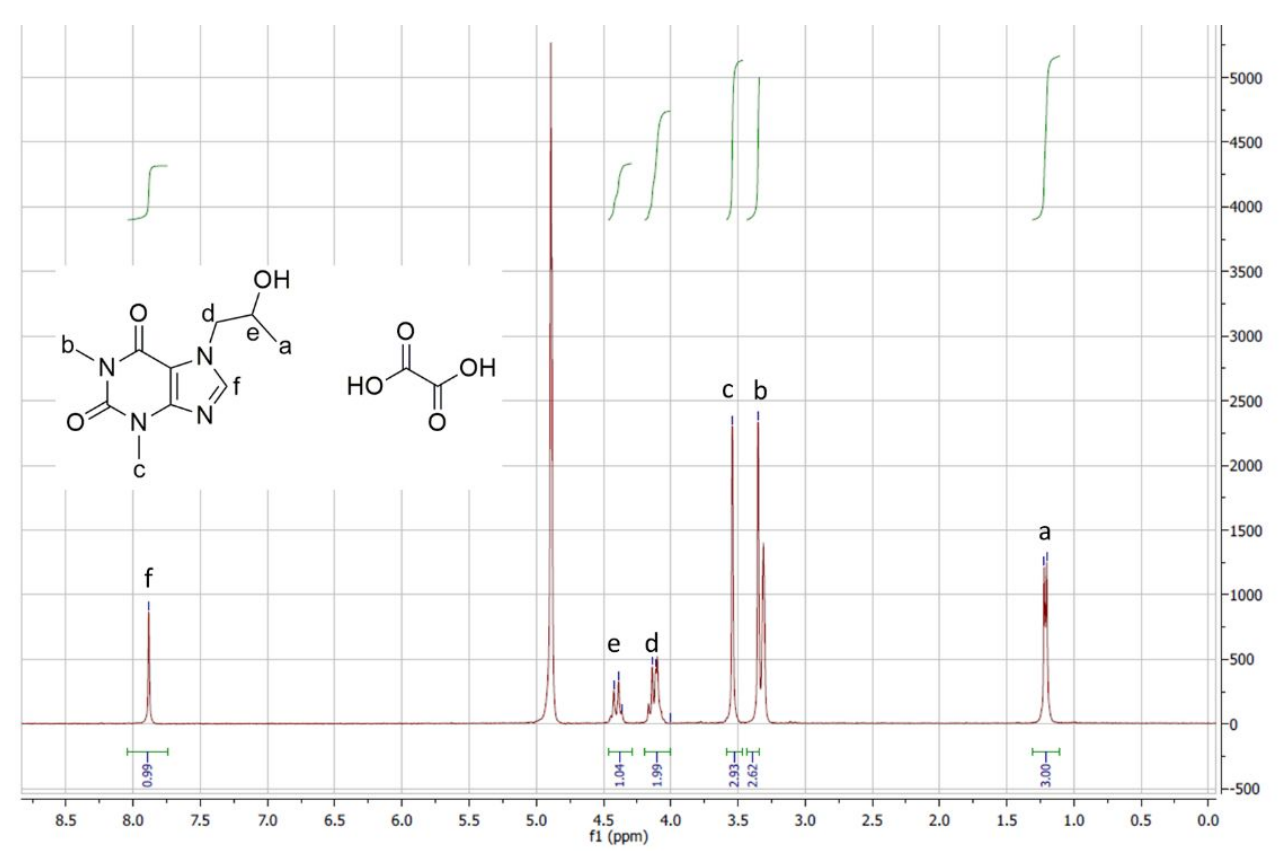

Figure S 1. ${ }^{1} \mathrm{H} N M R$ of the cocrystal obtained between PXL and OA in MeOD. Hydrogens of OA were not detected by NMR in MeOD. 


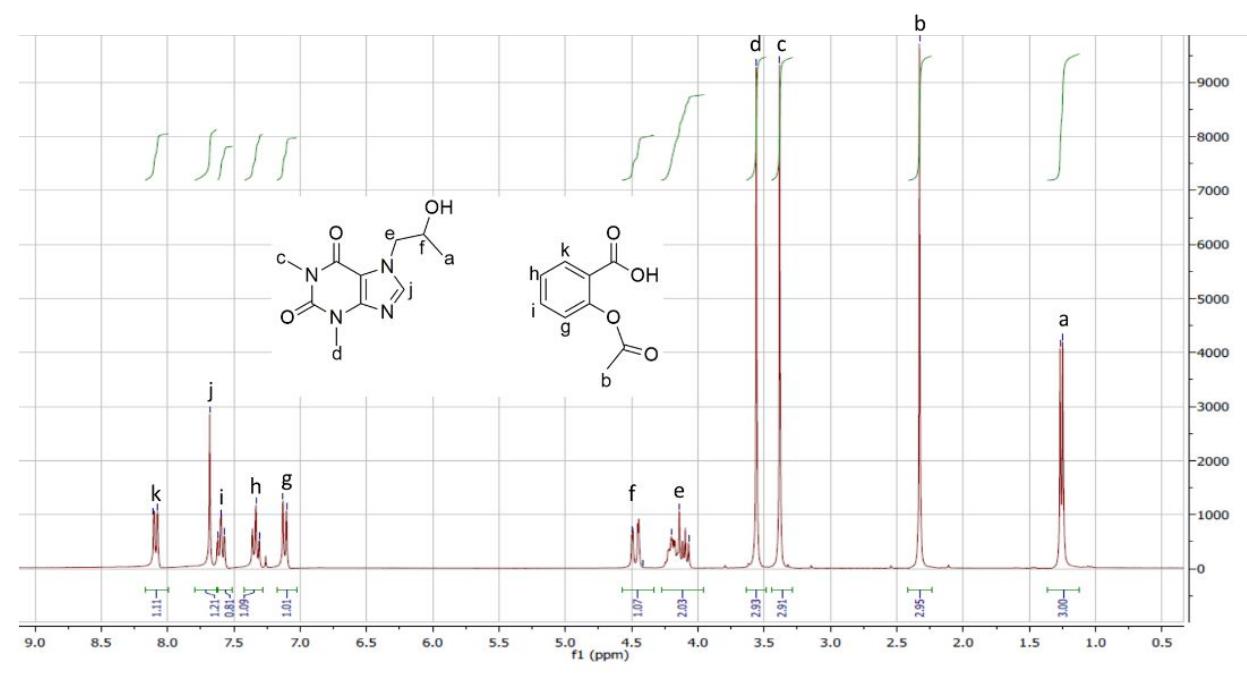

Figure S 2. ${ }^{1} \mathrm{H} N M R$ of the cocrystal between $P X L$ and $A A$ in $C D C l$.

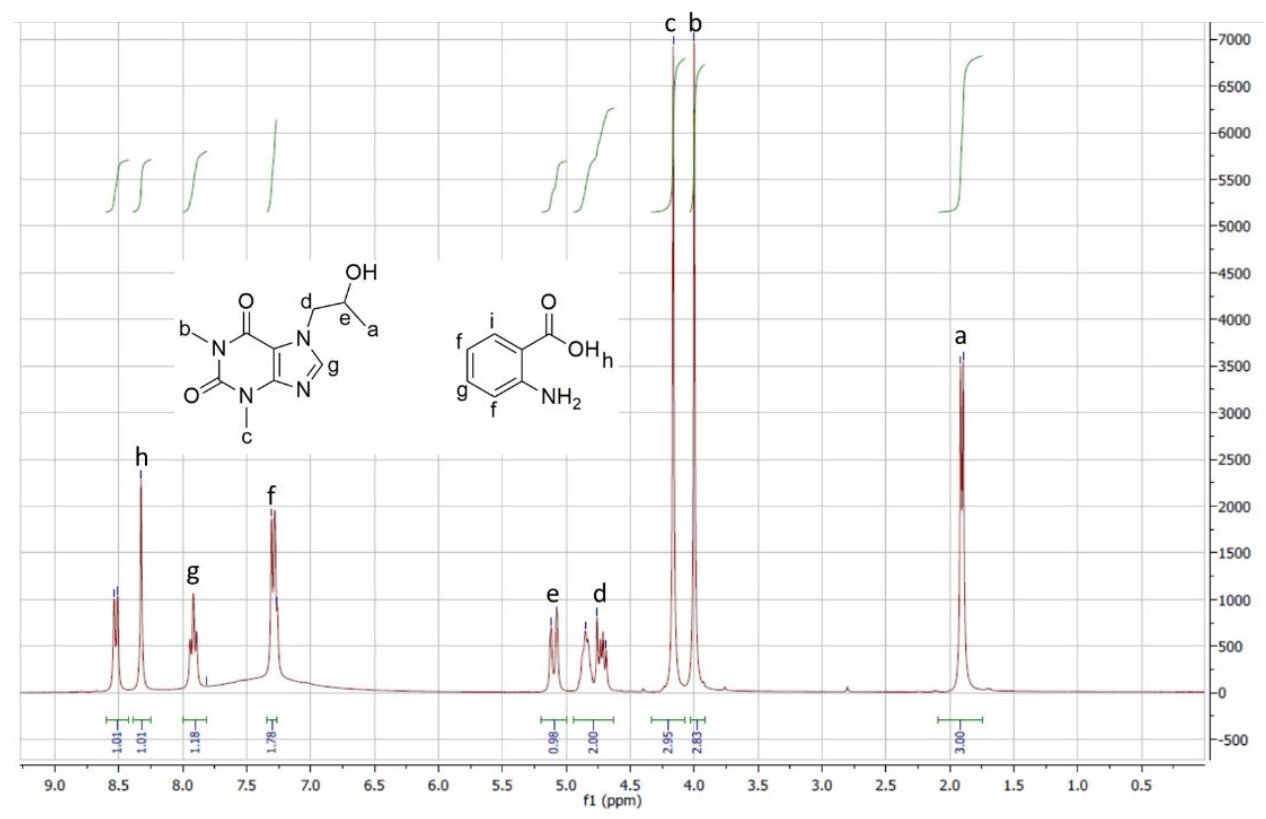

Figure S $3 .{ }^{1} \mathrm{H} N M R$ of the cocrystal between $P X L$ and Ant in $\mathrm{CDCl}_{3}$. 


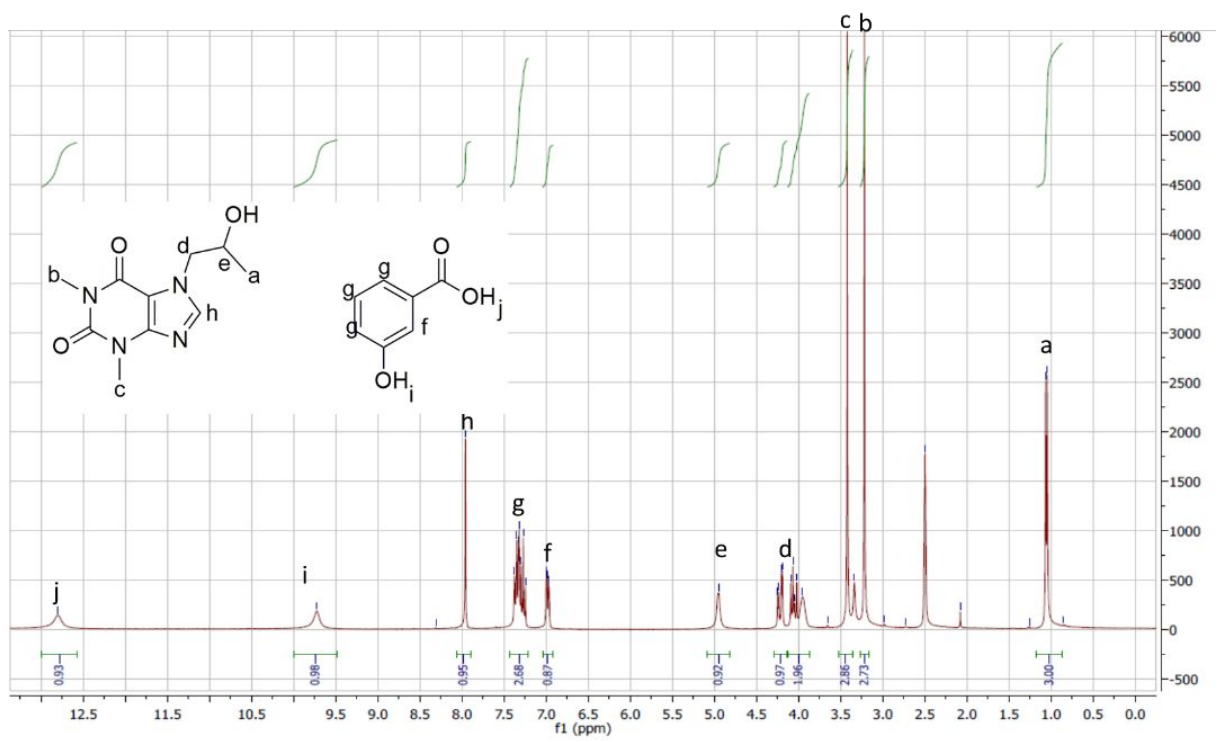

Figure S 4. ${ }^{1} \mathrm{H} N M R$ of the cocrystal between $\mathrm{PXL}$ and $\mathrm{HBA}$ in $\left(\mathrm{CD}_{3}\right)_{2} \mathrm{SO}$.

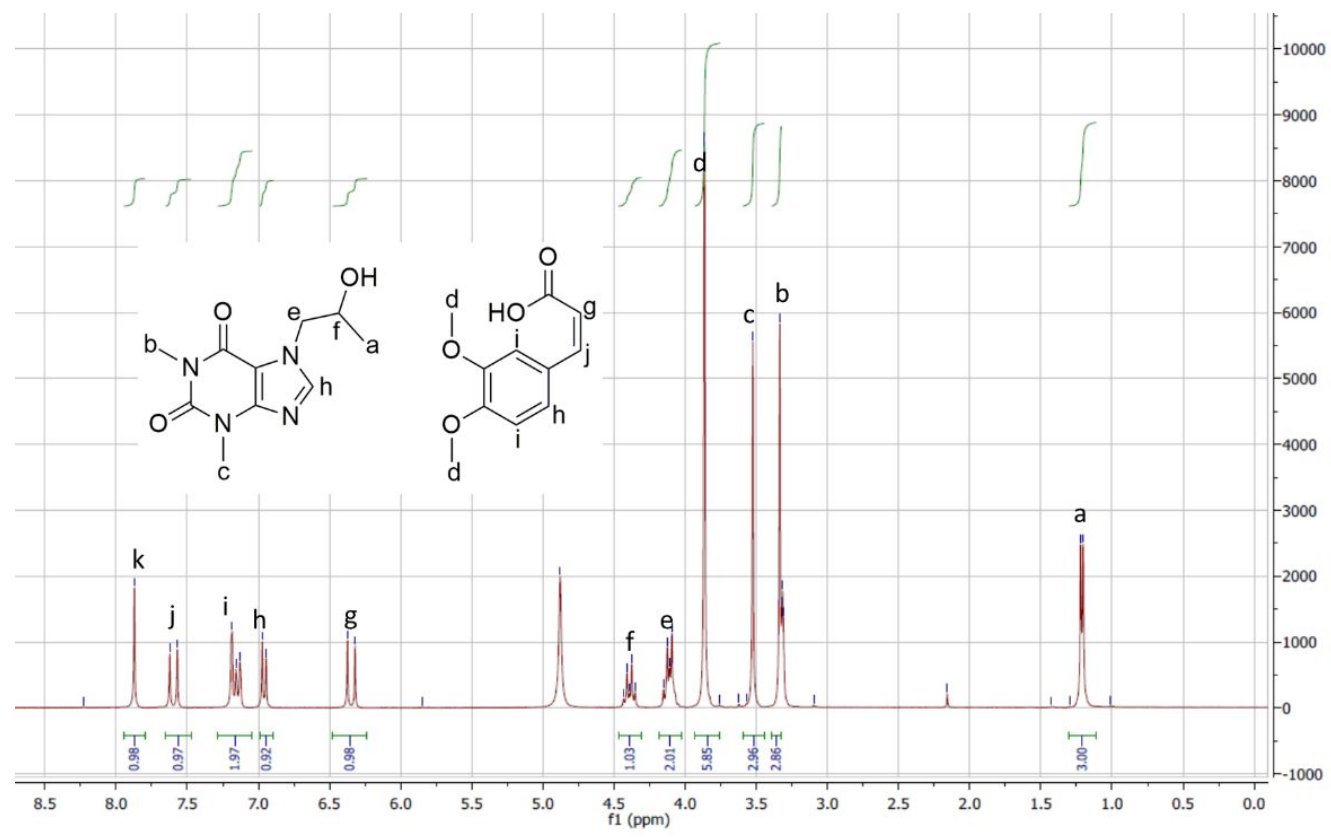

Figure S 5. ${ }^{1} \mathrm{H} N M R$ of the cocrystal between PXL and DMCA in MeOD. 


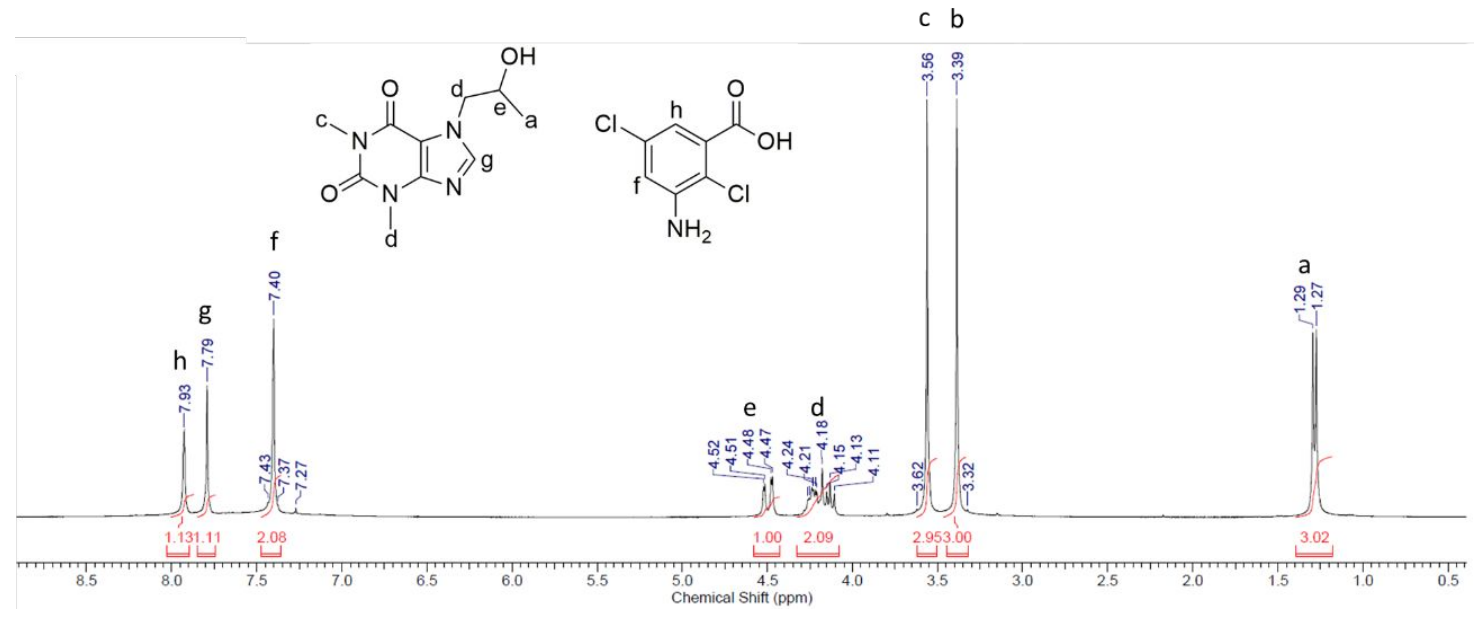

Figure S 6. ${ }^{1} H$ NMR of the cocrystal obtained between PXL and DCIBA.

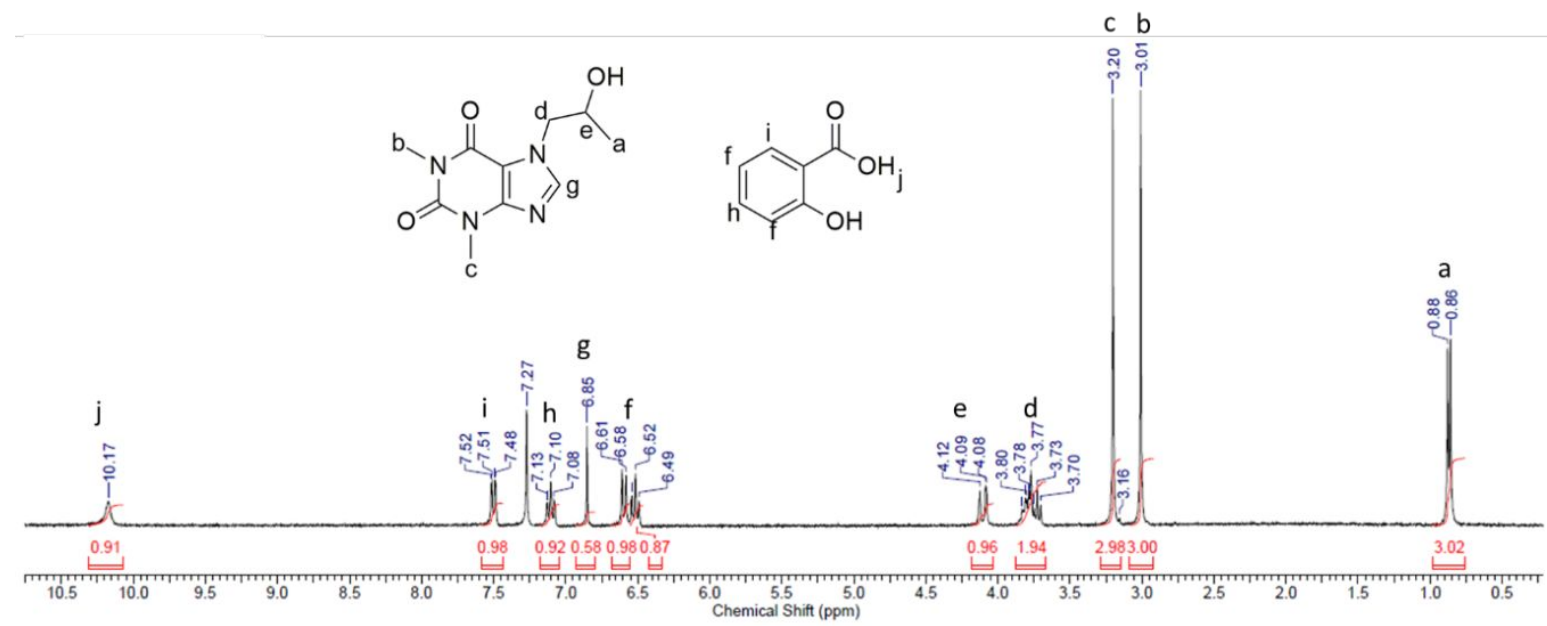

Figure S 7. ${ }^{1} H N M R$ of the cocrystal obtained between PXL and SA. 

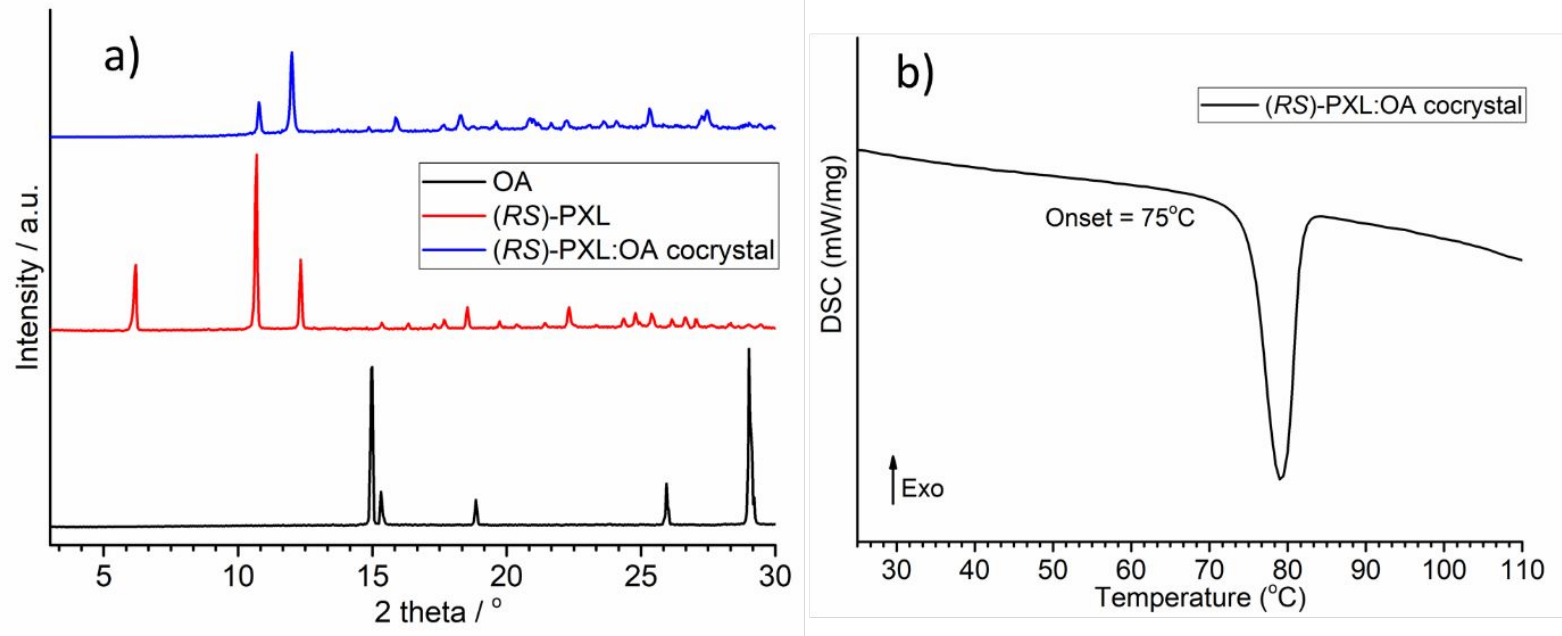

Figure S 8. a) XRPD pattern of the starting material (RS)-PXL, Oxalic acid (OA) and (RS)-PXL:OA cocrystal and b) DSC plot of the obtained cocrystal.
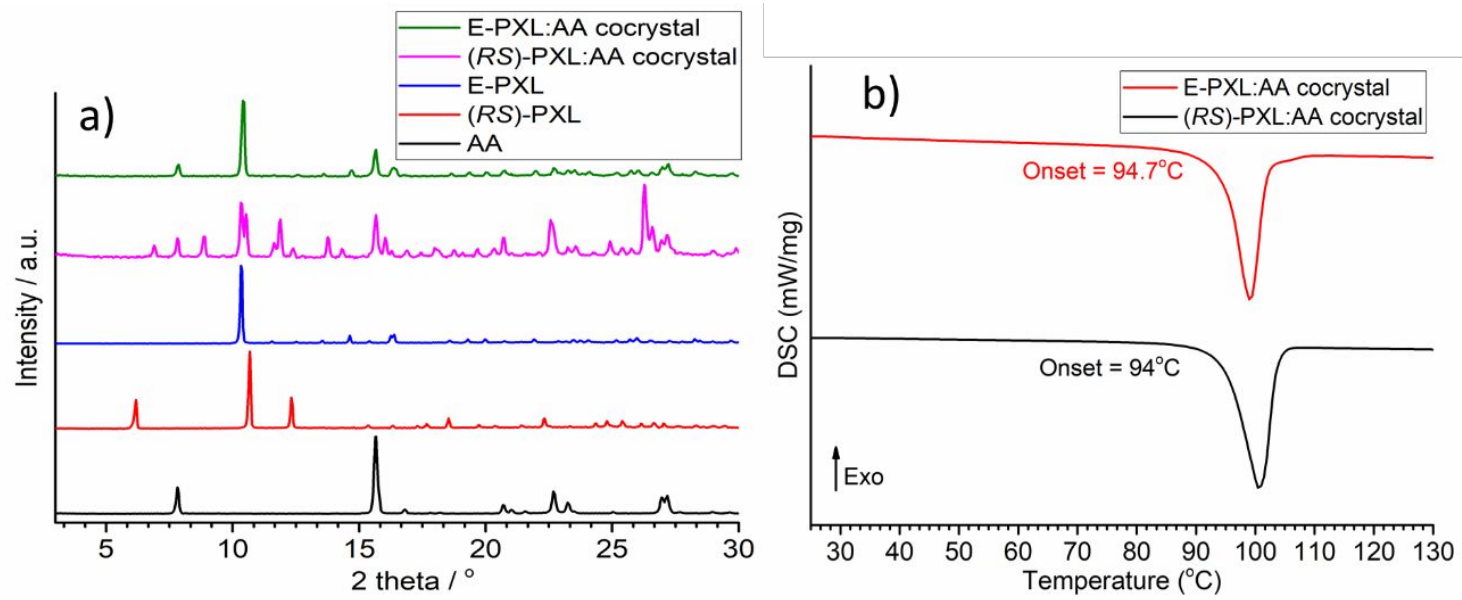

Figure S 9. a) XRPD pattern of (RS)-PXL, AA, E-PXL, (RS)-PXL:AA cocrystal and E-PXL:AA cocrystal in 1:1 molar ratio and $b)$ DSC plot of the obtained cocrystals. 

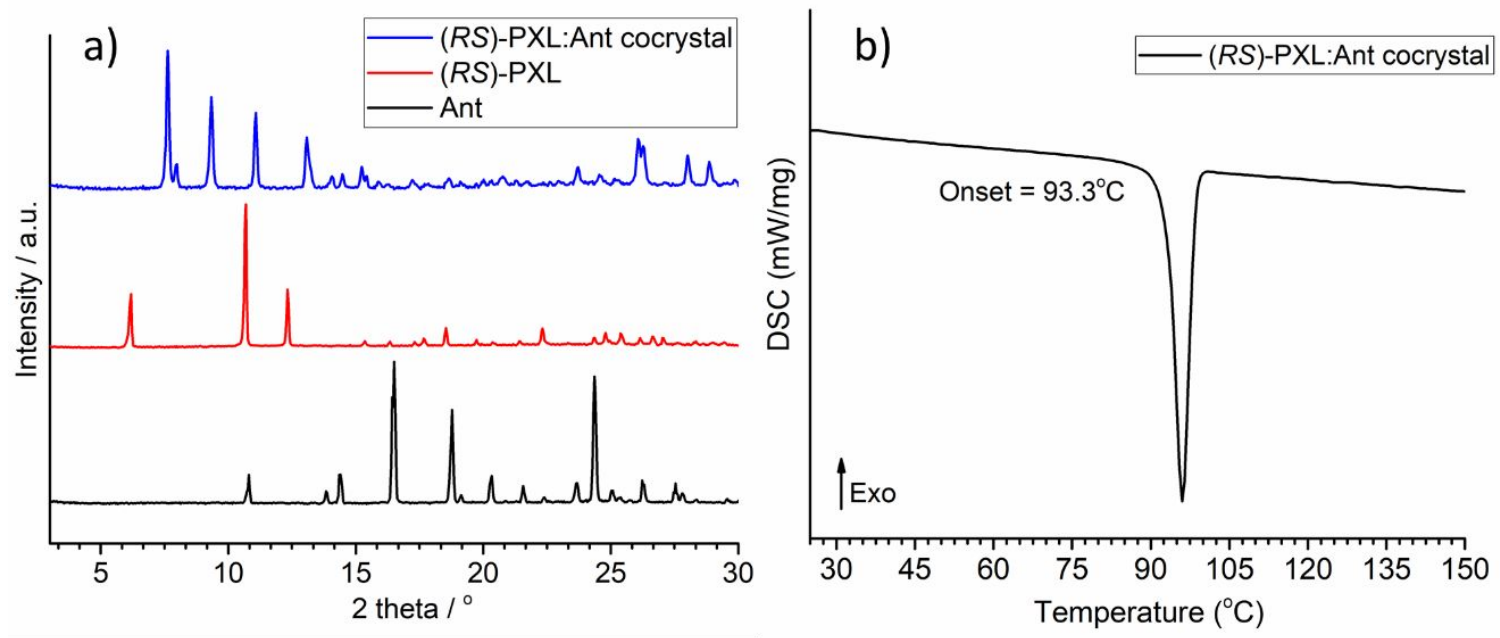

Figure S 10. a) XRPD pattern of (RS)-PXL, Ant and their mixture in 1:1 molar ratio. b) DSC plot for the obtained cocrystal.
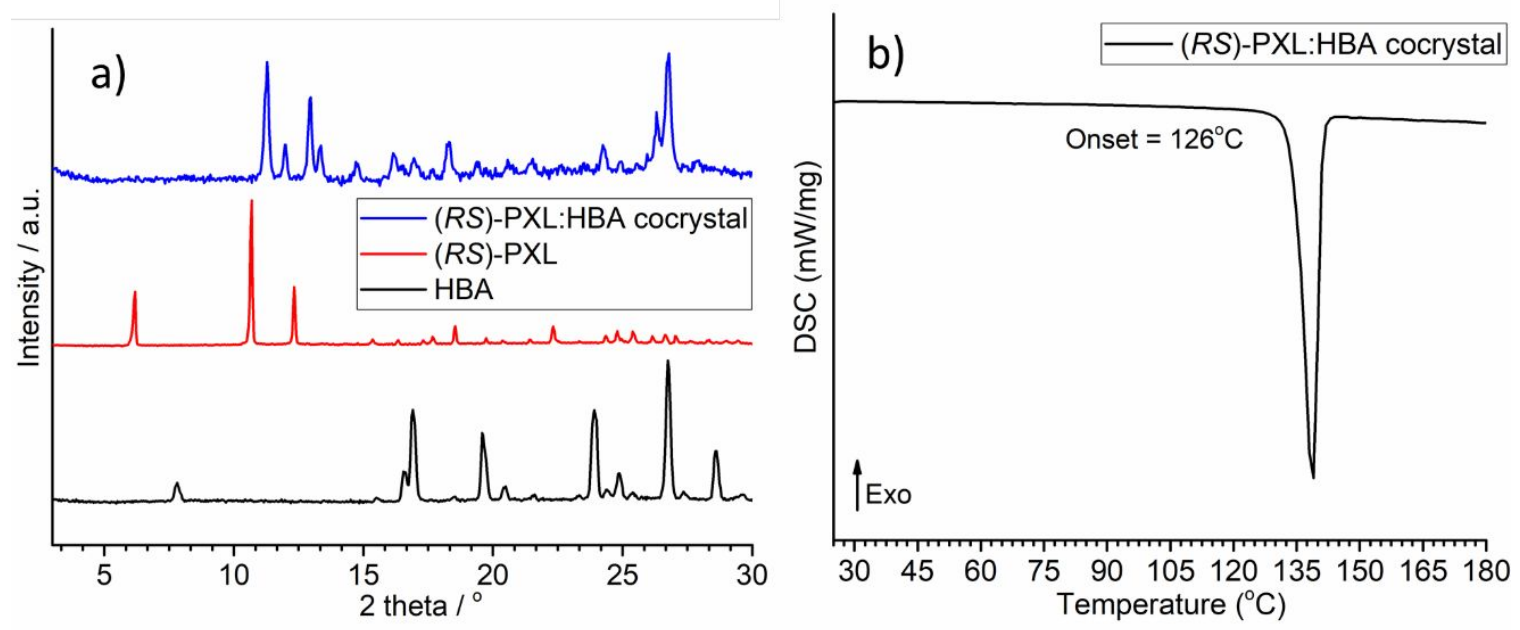

Figure S 11. a) Powder X-ray diffraction for (RS)-PXL, HBA and (RS)-PXL:HBA cocrystal and b) DSC melting curves of the cocrystal. 

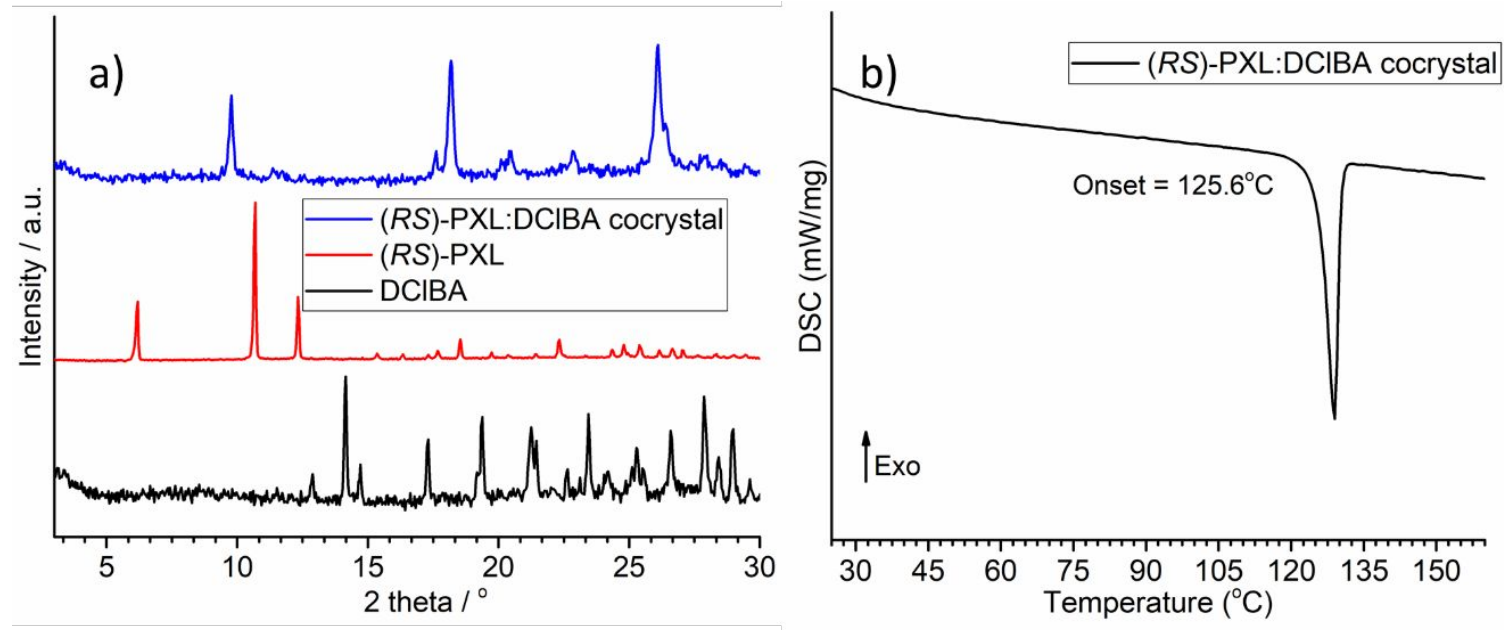

Figure S 12. a) XRPD for (RS)-PXL, DCIBA and PXL-DCIBA cocrystal and b) DSC melting curve of the new cocrystal form.
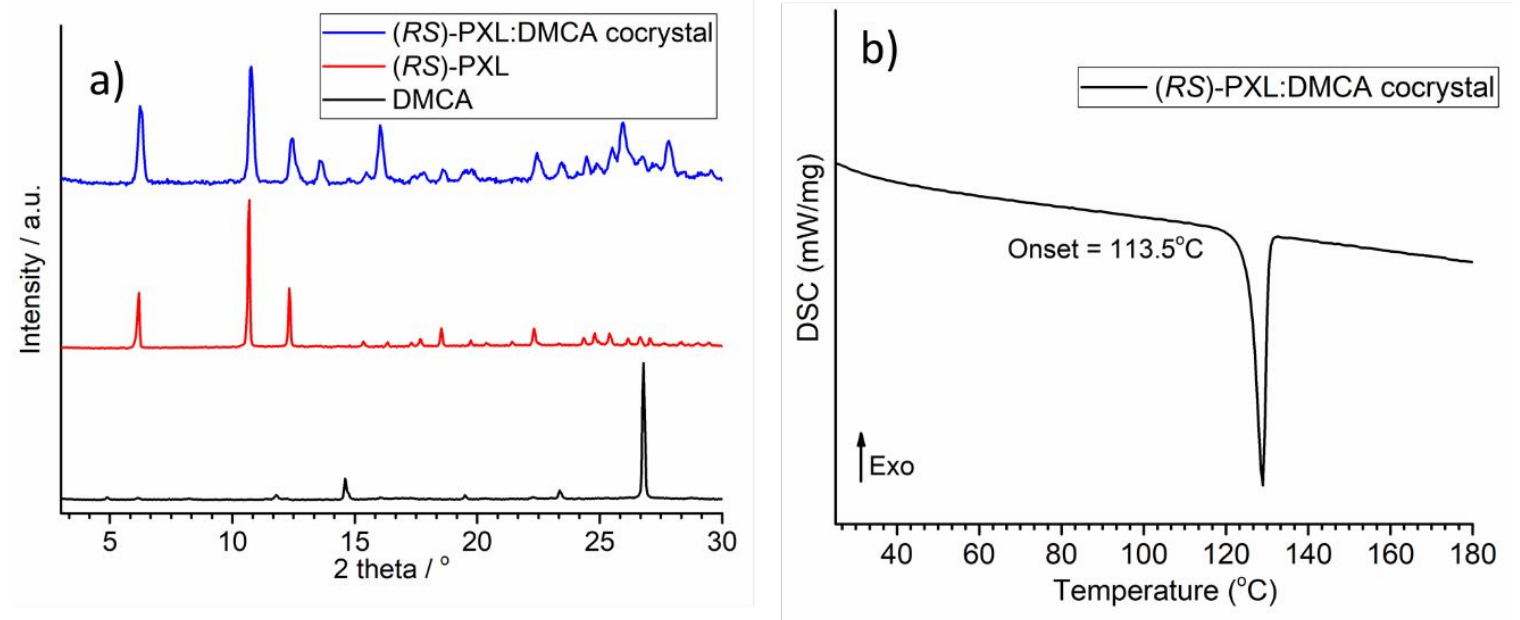

Figure S 13. a) XRPD for (RS)-PXL, DMCA and (RS)-PXL-DMCA cocrystal and b) DSC melting curve of the obtained cocrystal. 


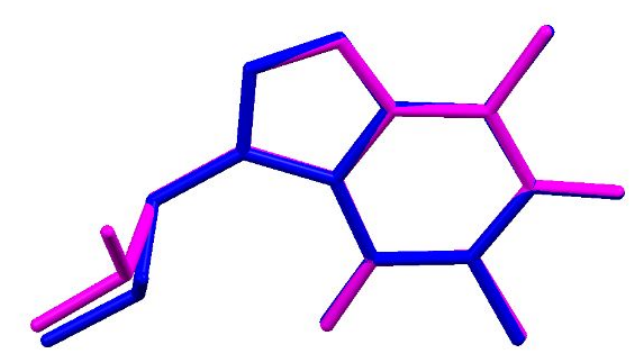

Figure S 14. Conformational similarity between the PXL molecules from the asymmetric unit of Form-H (Blue) and Form-A2 (Pink).

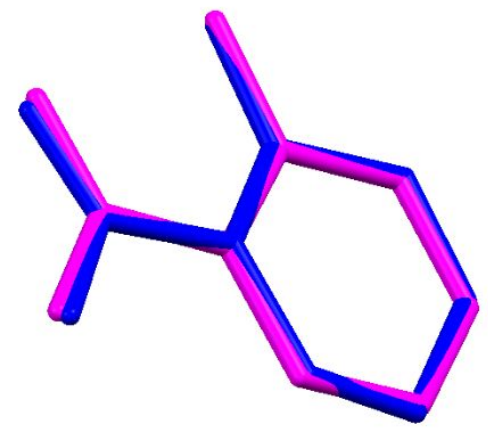

Figure $S$ 15. Conformational similarity between the SA molecules from the asymmetric unit of Form-H (Blue) and Form-A2 (Pink). 


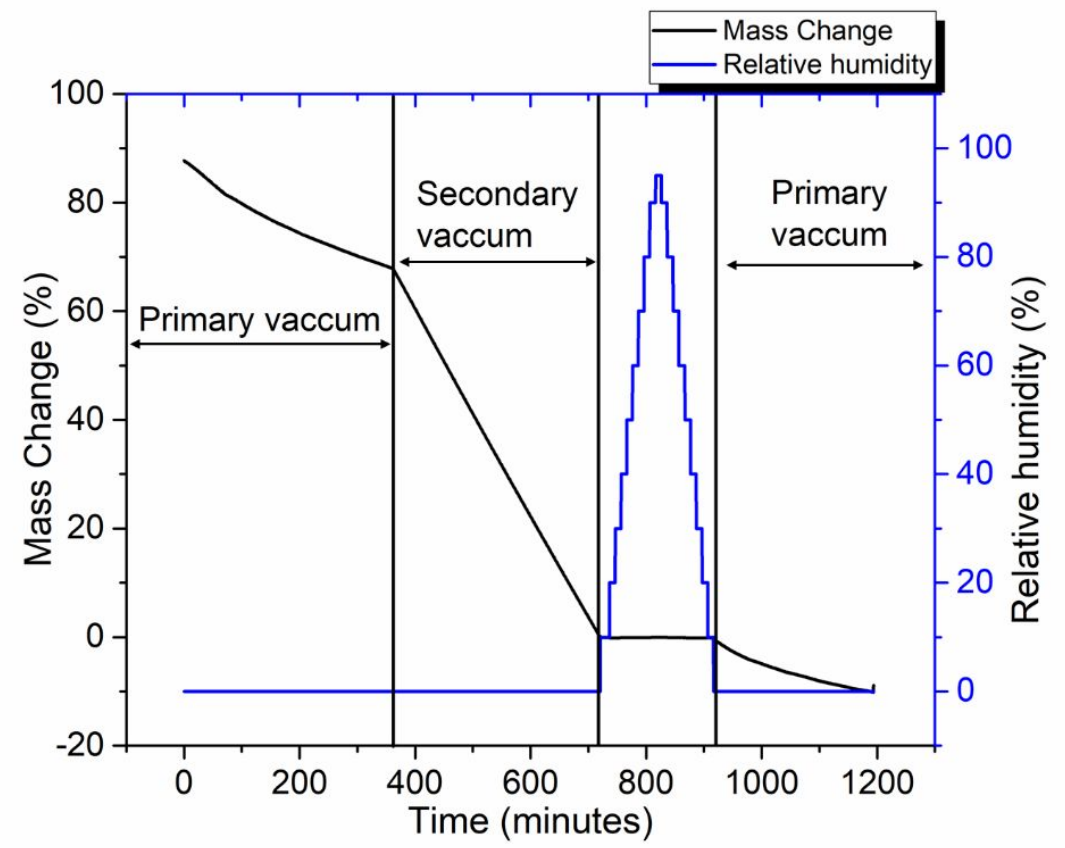

Figure S 16. Sorption-desorption cycle performed at $25{ }^{\circ} \mathrm{C}$ for SA with DVS vacuum. Mass change $(\%)$ is referred to the mass at the end of the first drying step.

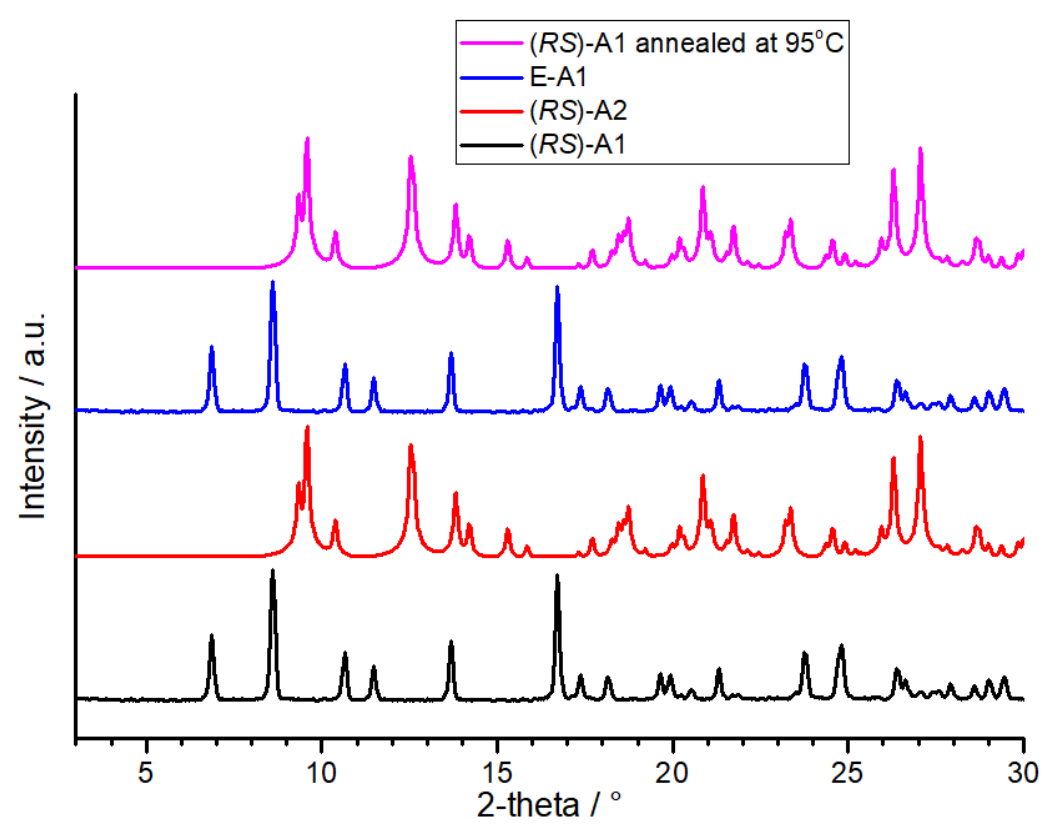

Figure S 17. XRPD patterns of (RS)-A1, (RS)-A2, E-A1 and the obtained solid after annealing (RS)A1 at $950 C$. 


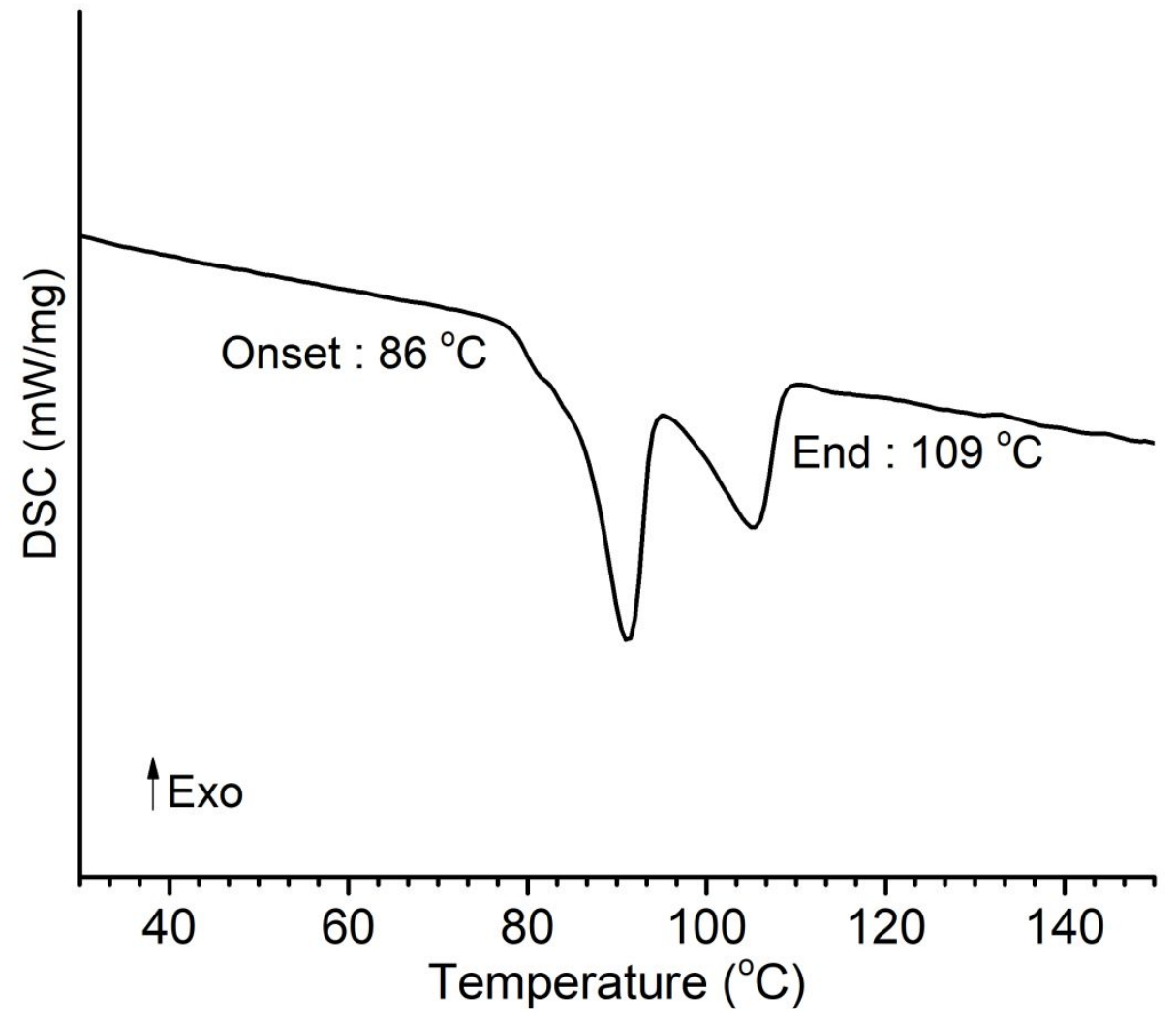

Figure S 18. DSC of the anhydrous form A3. 


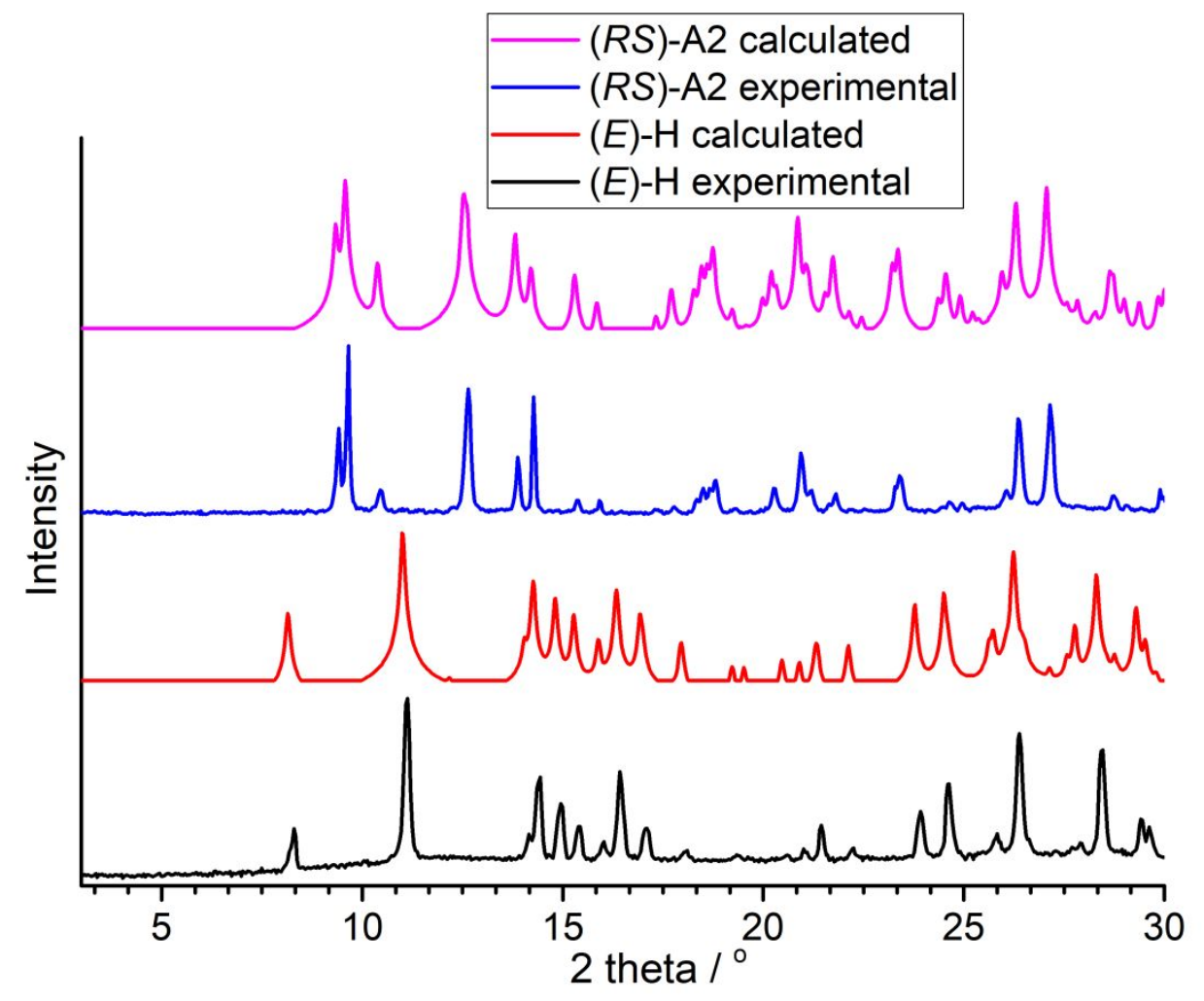

Figure S 19. Experimental and calculated patterns of (E)-H and (RS)-A2 respectively. 